\title{
Optical Bench Analysis of 2 Depth of Focus Intraocular Lenses
}

\author{
Andreas F. Borkenstein ${ }^{a} \quad$ Eva-Maria Borkenstein ${ }^{a}$ Holger Luedtke ${ }^{b}$ \\ Ruediger Schmid ${ }^{\mathrm{b}}$ \\ aBorkenstein \& Borkenstein, Private Practice at Privatklinik der Kreuzschwestern Graz, Graz, Austria; ${ }^{\mathrm{b}}$ Accuratis, \\ Practice for Refractive Eye Surgery, Ulm, Germany
}

\section{Keywords}

Enhanced depth of focus - Optical bench analysis .

Modulation transfer function · Strehl · Wavefront

\begin{abstract}
Background: The aim of the study was to analyze the objective optical properties of 2 enhanced depth of focus (EDoF) intraocular lenses (IOLs) using optical bench analysis. Methods: This experimental study investigates 2 new EDoF IOLs, the Alcon AcrySof IQ Vivity and the Bausch \& Lomb LuxSmart Crystal, on the optical bench, using OptiSpheric IOL PRO2 (Trioptics, Germany) in order to assess the optical quality according to ISO 11979 with ISO-2 Cornea. IOLs (power 22.0 D) were evaluated regarding modulation transfer function (MTF) at $50 \mathrm{lp} / \mathrm{mm}$ and Strehl ratio (SR) using a 3.0- $\mathrm{mm}$ and a $4.5-\mathrm{mm}$ aperture. In addition, wavefront measurements were obtained using WaveMaster ${ }^{\circledR}$ IOL 2 device (Trioptics, Germany), and USAF targets were analyzed. Results: Centered: the MTF (mean) at $50 \mathrm{lp} / \mathrm{mm}$ (AcrySof IQ Vivity/ LuxSmart Crystal) with $3.0 \mathrm{~mm}$ aperture was 0.250/0.257 and with $4.5 \mathrm{~mm}$ aperture 0.202/0.243. The SR (mean) with 3.0 $\mathrm{mm}$ aperture was $0.261 / 0.355$ and with $4.5 \mathrm{~mm}$ aperture $0.176 / 0.206$. Decentered by $1 \mathrm{~mm}$ : the MTF (mean) at $50 \mathrm{lp} /$ $\mathrm{mm}$ (AcrySof IQ Vivity/LuxSmart Crystal) with $3.0 \mathrm{~mm}$ aper-
\end{abstract}

ture was 0.266/0.247 and with $4.5 \mathrm{~mm}$ aperture 0.126/0.215. The SR (mean) with $3.0 \mathrm{~mm}$ aperture was $0.272 / 0.234$ and with $4.5 \mathrm{~mm}$ aperture $0.133 / 0.183$. Tilted by 5 degree: the MTF (mean) at $50 \mathrm{lp} / \mathrm{mm}$ (AcrySof IQ Vivity/LuxSmart Crystal) with $3.0 \mathrm{~mm}$ aperture was $0.221 / 0.360$ and with $4.5 \mathrm{~mm}$ aperture $0.214 / 0.229$. The SR (mean) with $3.0 \mathrm{~mm}$ aperture was $0.232 / 0.428$ and with $4.5 \mathrm{~mm}$ aperture $0.225 / 0.229$. The simulated visual function using USAF test targets showed corresponding qualitative results. Wavefront measurements proved a complex optical design. Higher order aberrations in the central part of the optics were modulated up to the 10th order to enhance the range of functional vision to near distance, leaving the peripheral parts of the optics aberration free or as aberration correcting. Conclusion: The diversity of EDOF IOLs, their optics, and their respective impact on the vision quality must be understood in order to select the appropriate IOL in each individual case. This analysis of new, innovative IOL optics based on increased negative spherical aberration may help the ophthalmic surgeon to select the IOL which meets the individual requirements of the patient for best postoperative outcomes. It seems that there is no perfect IOL that is equally suitable for all patients, but the right choice is an individual, customized approach dealing with patients' expectations.

(c) 2021 The Author(s)

Published by S. Karger AG, Basel karger@karger.com www.karger.com/bmh

Karger $\stackrel{\text { ' }}{5}$

GOPEN ACCESS
(C) 2021 The Author(s)

Published by S. Karger AG, Basel

This is an Open Access article licensed under the Creative Commons Attribution-NonCommercial-4.0 International License (CC BY-NC) (http://www.karger.com/Services/OpenAccessLicense), applicable to the online version of the article only. Usage and distribution for commercial purposes requires written permission.
Correspondence to:

Andreas F. Borkenstein, crustalith@gmx.at 


\section{Introduction}

Since the first intraocular lens (IOL) implantation by Harold Ridley in 1949, IOLs have continued to evolve. The materials, design, and stability of the IOLs have been continuously improved, always with the aim of improving visual acuity and thus the quality of life postoperatively, while at the same time reducing possible complications or undesirable side effects. Monofocal IOLs are those commonly implanted globally, but the number of premium lenses available from various manufacturers continues to grow annually. The main reasons for the dominance of monofocal IOLs appear to be the consistent and easily measured outcomes associated with their use, as well as the reduced risk of dysphotopsia (and therefore the need for IOL exchange) and also their reduced costs attached to their use [1]. In case of dissatisfaction, IOL exchange can be a challenging treatment option [2].

However, monofocal IOLs do not offer the levels of spectacle independence demanded by increasing numbers of patients due to our changing lifestyle. An alternative is the use of monofocal lenses targeting emmetropia in 1 eye and slight myopia in the other to achieve monovision as the desire for independence from glasses is becoming increasingly important.

Most multifocal IOLs, such as bi- and trifocal IOLs, generate multiple focal points or diffraction patterns to sharpen an object on the retina for multiple focal lengths $[3,4]$. With these IOLs, centering of the IOL is particularly important, as a decentered lens can cause disturbing lower and higher order aberrations (HOAs) [5-7]. Dysphotopsia and reduced contrast sensitivity can significantly impair the optical quality of vision and therefore the overall satisfaction of patients [8-12]. In order to close the gap between monofocal and multifocal IOLs and to combine the advantages of both systems, so-called monofocal-plus IOLs have been developed. These have become increasingly popular options in the intermediate sector and do have the potential to provide an extended range of vision (ERV) with far-point performance [13].

This new technology should give an ERV with the farpoint performance and photic phenomena comparable to that of monofocal IOL patients.

The first ERV or enhanced depth of focus (EDoF) lenses used diffractive echelette to extend the depth of field and create a wider range of desired, useful uncorrected vision (intermediate range). The American Academy of Ophthalmology has defined the criteria to facilitate and standardize the classification of an IOL as an "EDoFIOL," as it was a heterogeneous class of IOLs [14-17]. Ac- cording to this, an EDoF lens should always be compared with a monofocal IOL. Among the proposed criteria, the best-corrected distance visual acuity should be comparable to the monofocal control lens, while the distancecorrected intermediate visual acuity should be better than that of the control lens. The defocus curve should show a defocus at $66 \mathrm{~cm}$ where visual acuity is 0.2 LogMAR or better in $50 \%$ of patients.

A differentiation is made between so-called pure EDoF lenses and hybrid-multifocal EDoF lenses. While pure EDoF IOLs are based on spherical aberrations (SAs) (Alcon AcrySof IQ Vivity and Bausch \& Lomb LuxSmart) or use the pinhole effect (e.g., Morcher XtraFocus or AcuFocus IC-8), hybrids are divided into refractive (e.g., Acunex Vario, SAV Lucidis, and Sifi MiniWell) and diffractive (e.g., Tecnis Symfony and Zeiss LARA) IOLs and a further subgroup of refractive-diffractive IOLs (e.g., SAV-IOL Harmonis).

By this definition of the American Task Force, the novel monofocal + IOLs Alcon AcrySof IQ Vivity and the Bausch \& Lomb LuxSmart Crystal IOL can be defined as EDoF IOLs.

The purpose of this article was to compare 2 novel IOLs - the Alcon AcrySof IQ Vivity IOL and the B\&L LuxSmart IOL - with respect to their performance on the optical bench. Using the definition of the American Task Force, these "monofocal plus" IOLs, with optics based on increased negative SA, may be defined as EDOF IOLs. Objective and independent evaluation was ensured by third parties' measurements and statistical analysis [18].

\section{Methods}

Optical Quality Measurement

Optical Metrology Device

The OptiSpheric IOL PRO2 (Trioptics GmbH, Wedel, Germany) was used to measure the optical performance of the study IOLs, which follows the guidelines of the International Standard Organization. The OptiSpheric IOL PRO2 consists of a polychromatic light source (light-emitting diode), spectral filters, a test object (i.e., 2 perpendicular slits), a collimator, an IOL holder filled with a balanced salt solution, a microscope objective, and a charge-coupled device (CCD) camera. The test pattern was illuminated by a collimator and imaged by the IOL under test onto the CCD camera. This arrangement was used to assess the optical transfer function.

The OptiSpheric IOL PRO2 derives the optical quality parameters (modulation transfer function $[\mathrm{MTF}]$ ) from the Fourier transform of the line spread function projected by the lens under test. The optical transfer function components were assessed using a spectral filter that simulates the photopic luminosity function established by the Commission Internationale de l'Éclairage. All measurements were performed using an ISO-2 model cornea with $+0.28 \mu$ ISO 11979/2), lens placement in situ (balanced salt solu- 
tion), and a temperature of $35^{\circ}$. Different aperture diameters were used to simulate pupil size.

\section{MTF and Strehl Ratio}

In order to assess the optical quality of an IOL and to make it comparable with others, the optical quality parameters for aperture sizes $3.0 \mathrm{~mm}$ and $4.5 \mathrm{~mm}$ at the IOL plane were assessed. MTF and Strehl ratio (SR) values, rf "Autofocus Scan" - as well as the USAF 1951 test targets "US Air Force 1951 resolution test chart images" as qualitative simulation, were analyzed. The MTF is a way of describing the contrast sensitivity of a lens system; for the human eye, it can be regarded as "visual performance." MTF is the imaging performance of a lens at several spatial frequencies in tangential and sagittal directions. In our evaluation, we used MTF tan and MTF sag values to calculate the mean value. Since the SR accounts for all small oscillations that occur on the MTF curve, it reflects the overall optical performance. The SR is the ratio of the sum of all measured MTF values divided by the sum of all the MTF values. Accordingly, a perfect IOL would have an SR of 1.0. Therefore, the smaller the SR value, the poorer the optical quality. In order to simulate photopic and mesopic pupillary conditions, 2 aperture sizes ( 3.0 and $4.5 \mathrm{~mm}$ ) were used for each measurement.

\section{USAF 1951 Resolution Test Chart}

We used in situ measurement conditions $(\mathrm{NaCl}$ solution with cornea [SA 0. $28 \mu \mathrm{m}$ ]) to evaluate USAF resolution targets (ISO 11979). For this measurement procedure, a reticle is selected, and the focus of the IOL is sought by moving the Mic 10x attached to the Z-stage in such a way that a clearly imaged cross can be seen on the camera image. A diopter scan is performed at this position. During this scan, the measuring cross is defocused. The Z-stage method is used to determine the best focus position. At this position, the shutter time is automatically adjusted so that the light intensity is approximately $75 \%$. Then the USAF target is swiveled in and the shutter time is optimized again. The first image is taken at this position (Nom_MTF). This image is taken in the best focus position for MTF measurement. With the USAF target still swiveled in, a gradient scan is then made to find the best image with this reticle (Nom_Gradient).

\section{Wavefront Measurements}

We assessed these 2 wavefront modulating IOLs on the optical bench to get objective information about the wavefront and power pattern under standardized conditions independent of the manufacturers' statements. Measurements were conducted by Trioptics $\mathrm{GmbH}$, Wedel, Germany, with the WaveMaster ${ }^{\circledR}$ IOL 2 device test bench, a high-resolution Shack-Hartmann sensor in reverse projection setup with a dynamic range up to 2,000 lambda $(\lambda)$, an accuracy of $\lambda / 20$ (RMS), and a reproducibility of $\lambda / 200$ (RMS). The WaveMaster ${ }^{\circledR}$ IOL 2 device complies with international standards, and all measurements were done in $\mathrm{NaCl}(n=1,337)$ and in room temperature with an in situ eye model according to ISO 11979. In this setting, the sample IOL is illuminated using a point laser light source produced by a collimator with fiber-coupled laser light input. We used a wavelength of $546 \mathrm{~nm}$ (ISO). With this wavelength, our results in $\lambda$ can be transformed to $\mu$ by calculating $\lambda \times 546 / 1,000$. The image of the IOL aperture is projected on a wavefront sensor consisting of a microlens array placed in front of a CCD camera. The measured wavefront corresponds to the aberrations generated by the IOL. Each focus spot position shift of the individual micro-

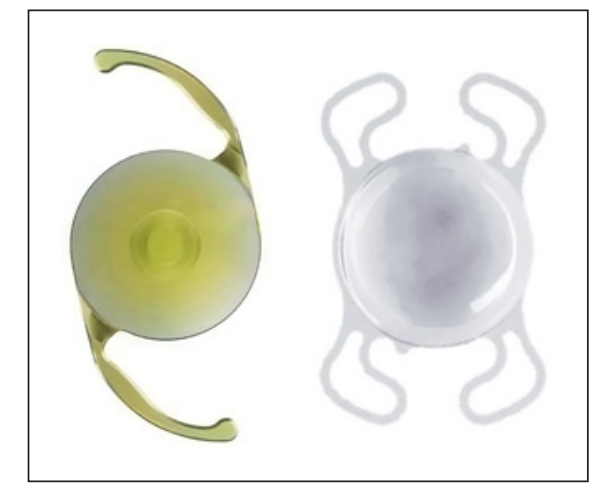

Fig. 1. AcrySof IQ Vivity and LuxSmart Crystal.

lens of the wavefront sensor's array is measured, and the resulting wavefront profile map is calculated and displayed. Thus, a continuous wavefront mapping of the IOL can be obtained over the entire aperture. Zernike polynomials up to the 10th order can be determined by means of the measured wavefront and describing the typical optical properties of each IOL.

\section{Intraocular Lenses}

We studied 2 IOL models, each having the same refractive power of +22.0 D (Fig. 1). AcrySof IQ Vivity DFT015 IOL (Alcon, Fort Worth, TX, USA) is a hydrophobic, single-piece acrylic IOL with an overall diameter of $13.0 \mathrm{~mm}$ and an optical diameter of $6.0 \mathrm{~mm}$ with a classic C-loop design. The hydrophobic acrylic material has a refractive index of 1.55 at $35^{\circ} \mathrm{C}$ and a low Abbe number of 37 . The aspheric design of the Vivity corrects $-0.20 \mu \mathrm{m}$ of primary SA.

The extension of the visual area to the intermediate distance is generated by a so-called X-Wave technology ${ }^{\mathrm{TM}}$ (nondiffractive wavefront-shaping technology). According to the manufacturer, the Vivity has 2 transition elements in the central $2.2 \mathrm{~mm}$ range. The first transition element stretches the wavefront, creating a continuous focus area. The light is stretched in both directions, that is, in the myopic and hyperopic directions. The light in the hyperopic direction is located behind the retina and would not be usable. Therefore, the second transition element moves the wavefront forward, shifting the light from the hyperopic direction to the myopic direction so that the entire light energy is used. The Vivity IOL generates the extended depth of field by means of the aspherical front lens surface and a spherical rear surface.

The LuxSmart Crystal (Bausch \& Lomb, Bridgewater, NJ, USA) is based on a 4-point fixation design. The hydrophobic, singlepiece acrylic IOL has an overall diameter of $10.0 \mathrm{~mm}$ and an optic diameter of $6.0 \mathrm{~mm}$ and is available with and without a violet filter. The lens design of this EDoF IOL is based on the so-called pure refractive optics principle. There is an EDoF center followed by a transition zone and a monofocal aberration neutral periphery. According to the manufacturer, the LuxSmart uses the 4th order SA for depth of field enhancement. With a combination of Z4-0 and Z6-0 with inverse sign, the greatest possible depth of field is supposed to be reached (approx. $1.75 \mathrm{dpt}$ according to the manufacturer) - this means that mathematically a sharp vision from $57 \mathrm{~cm}$ to infinity is possible. 


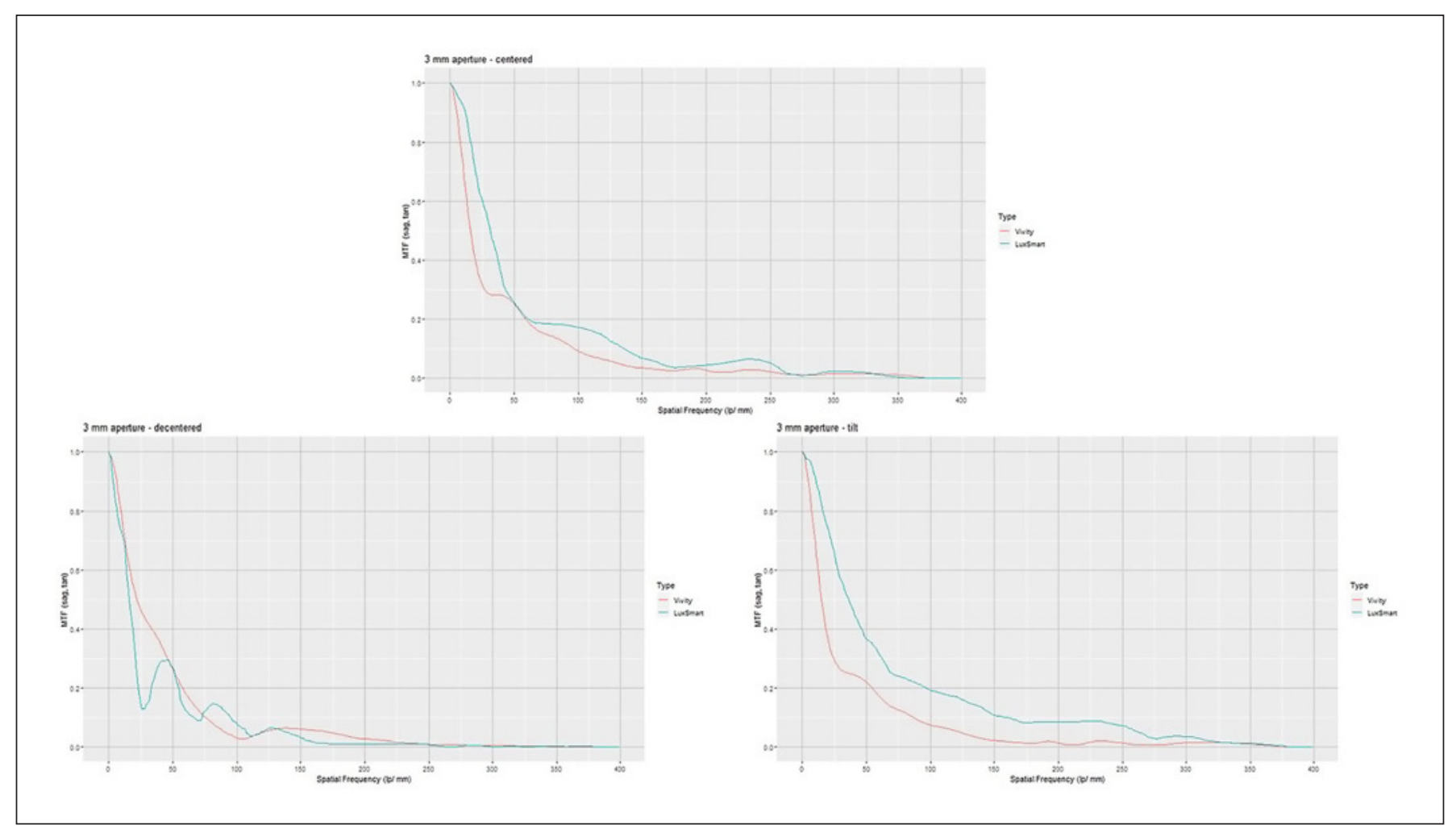

Fig. 2. MTF curves with $3.0 \mathrm{~mm}$ apertures. MTF, modulation transfer function.

\section{Statistics}

For the statistical evaluation of MTF and SR, an analysis of variance was performed with the factors IOL, MODE, and aperture and all interactions to degree 2 . As post hoc test a Tukey test was used. The significance level was set to 0.05 . The analysis was done with $\mathrm{R}$ version 3.6.1.

\section{Results}

\section{Optical Quality Assessment}

The MTF curves of all IOLs measured at the best focus through the 3.0-mm (Fig. 2) and 4.5-mm (Fig. 3) apertures are presented (Table 1). Centered: The MTF (mean) at $50 \mathrm{lp} / \mathrm{mm}$ (AcrySof IQ Vivity/LuxSmart Crystal) with $3.0 \mathrm{~mm}$ aperture was $0.250 / 0.257$ and with $4.5 \mathrm{~mm}$ aperture $0.202 / 0.243$. The SR (mean) with $3.0 \mathrm{~mm}$ aperture was $0.261 / 0.355$ and with $4.5 \mathrm{~mm}$ aperture $0.176 / 0.206$. Decentered by $1 \mathrm{~mm}$ : The MTF (mean) at $50 \mathrm{lp} / \mathrm{mm}$ (AcrySof IQ Vivity/LuxSmart Crystal) with $3.0 \mathrm{~mm}$ aperture was $0.266 / 0.247$ and with $4.5 \mathrm{~mm}$ aperture $0.126 / 0.215$. The SR (mean) with $3.0 \mathrm{~mm}$ aperture was $0.272 / 0.234$ and with $4.5 \mathrm{~mm}$ aperture $0.133 / 0.183$. Tilted by 5 degree: The MTF (mean) at $50 \mathrm{lp} / \mathrm{mm}$ (AcrySof IQ Vivity/LuxSmart Crystal) with $3.0 \mathrm{~mm}$ aperture was $0.221 / 0.360$ and with $4.5 \mathrm{~mm}$ aperture $0.214 / 0.229$. The SR (mean) with 3.0 $\mathrm{mm}$ aperture was $0.232 / 0.428$ and with $4.5 \mathrm{~mm}$ aperture $0.225 / 0.211$. Strehl findings were in accordance with the MTF results. This indicates, with certain limits, the corresponding expected retinal image quality (Table 1).

\section{USAF Test Charts}

The simulated visual function using USAF test targets showed partly corresponding qualitative results (Fig. 4, 5). Slight differences in brightness, halos, and ghosting were observed. With $3.0 \mathrm{~mm}$ aperture and lenses well centered, Vivity showed a sharp image but little brightness. LuxSmart USAF image was bright, yet somewhat blurred and had less contrast. For the IOLs centered and with $4.5 \mathrm{~mm}$ aperture, Vivity's image was slightly shaded and blurred but better than LuxSmart's image, which was not legible. For the IOLs decentered of $1 \mathrm{~mm}$ and with 3.0 $\mathrm{mm}$ aperture, Vivity showed less glare than LuxSmart. With IOLs decentered of $1 \mathrm{~mm}$ and with an aperture of 


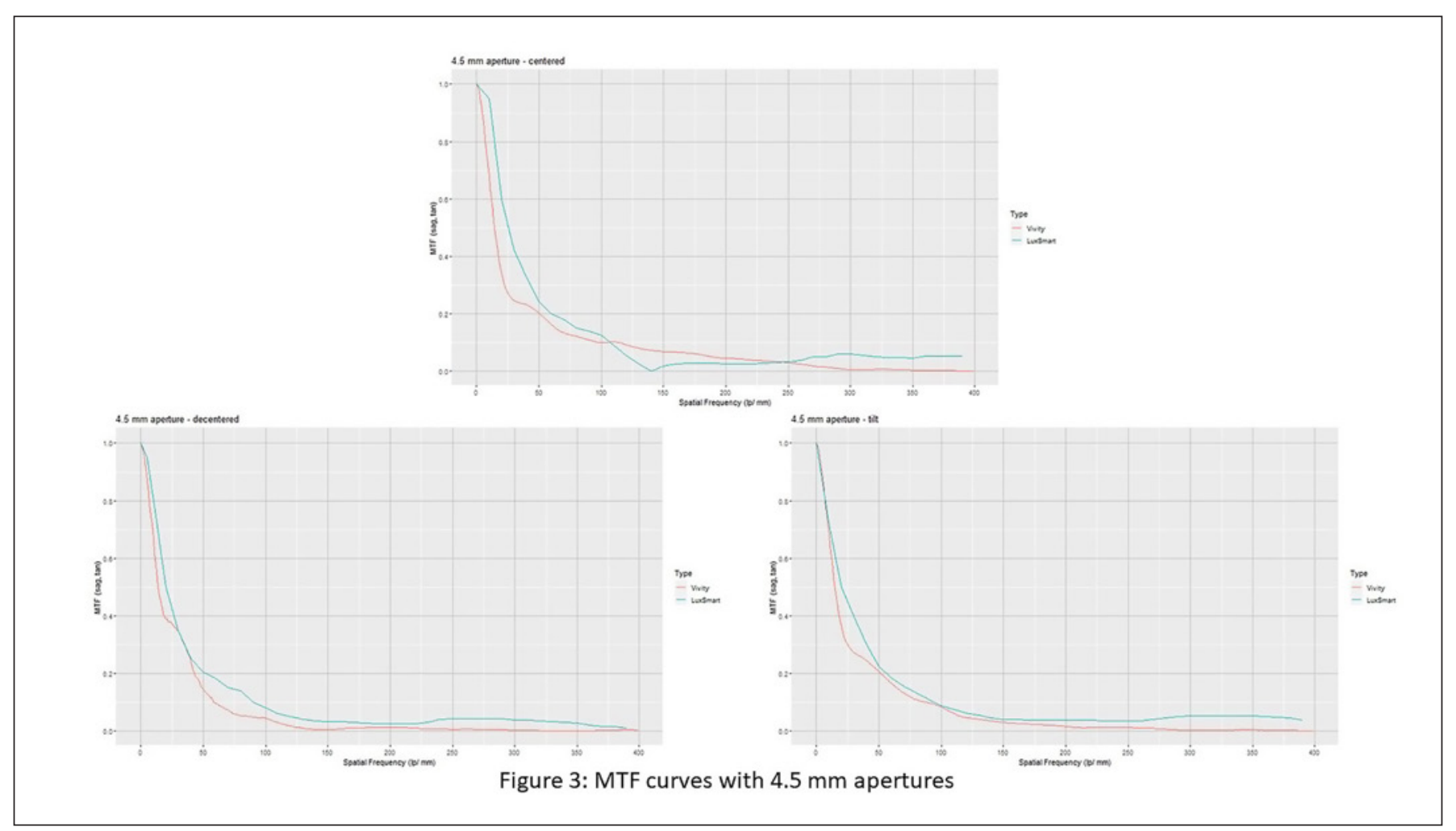

Fig. 3. MTF curves with $4.5 \mathrm{~mm}$ apertures. MTF, modulation transfer function.

Table 1. Data of MTF (50 lp/mm) and Strehl (mean) of the 2 IOLs

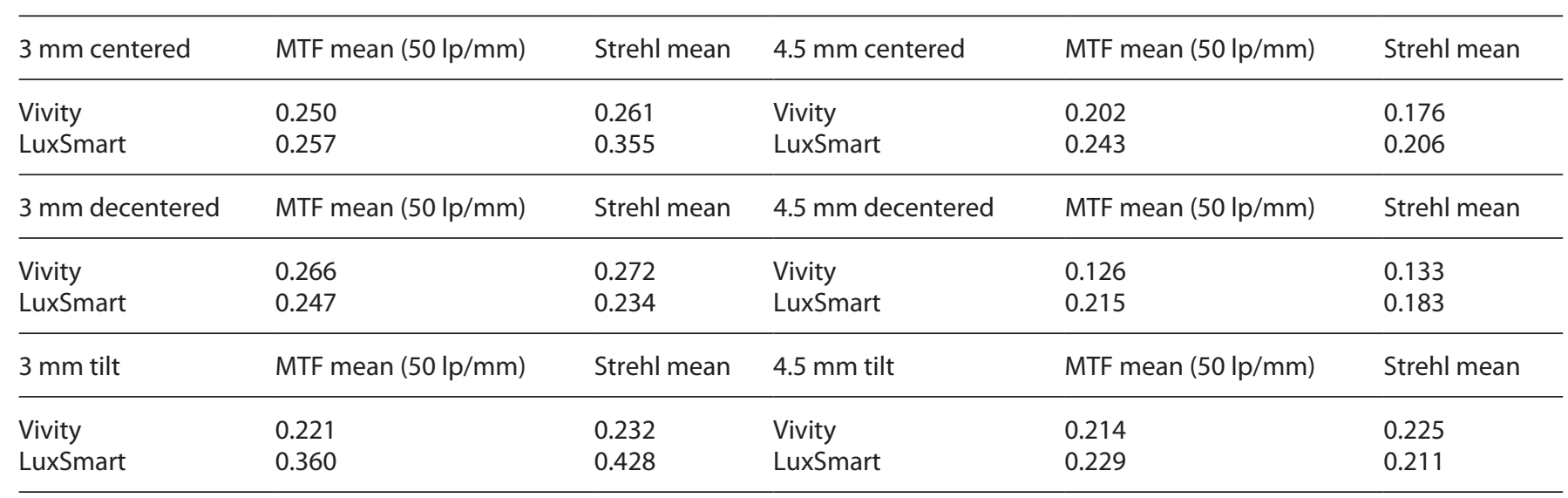

MTF, modulation transfer function; IOL, intraocular lens.

$4.5 \mathrm{~mm}$, Vivity performed better than LuxSmart, which image was blurred and, in some parts, not readable anymore. The analysis of the USAF test targets as a qualitative estimation of visual performance showed a fairly good image quality for the small aperture of $3 \mathrm{~mm}$ for both lenses and for all modifications of misalignment. LuxSmart showed considerable halos and ghosting, whereas a loss of brightness was seen for Vivity. These 
Fig. 4. USAF targets: Vivity (above) and LuxSmart (below) with $3.0 \mathrm{~mm}$ apertures (centered, decentered, and tilted).
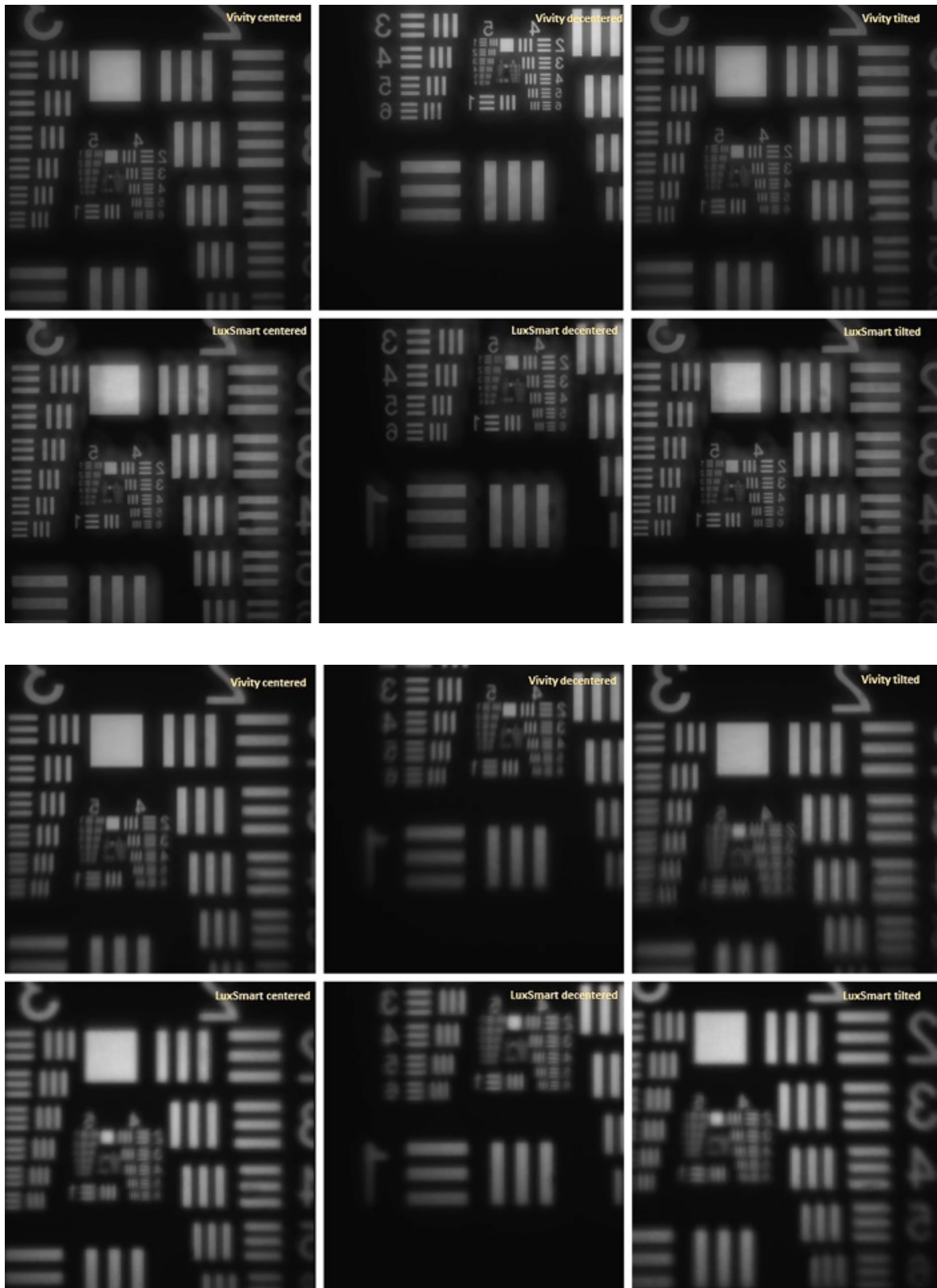

Fig. 5. USAF targets: Vivity (above) and LuxSmart (below) with $4.5 \mathrm{~mm}$ apertures (centered, decentered, and tilted).
Wavefront Results

A complex anterior surface wavefront modeling is used in both enhanced monofocal IOLs to smoothly modificate HOAs referring to the manufacturer's specifications (Fig. 6). HOAs were obtained up to the 10th order. Complex anterior surface wavefront modeling is employed in both of the enhanced monofocal IOLs to smoothly modify HOAs. The complex wavefront shaped IOLs could be measured with good accuracy and showed the expected increase and modulation of SA to produce 


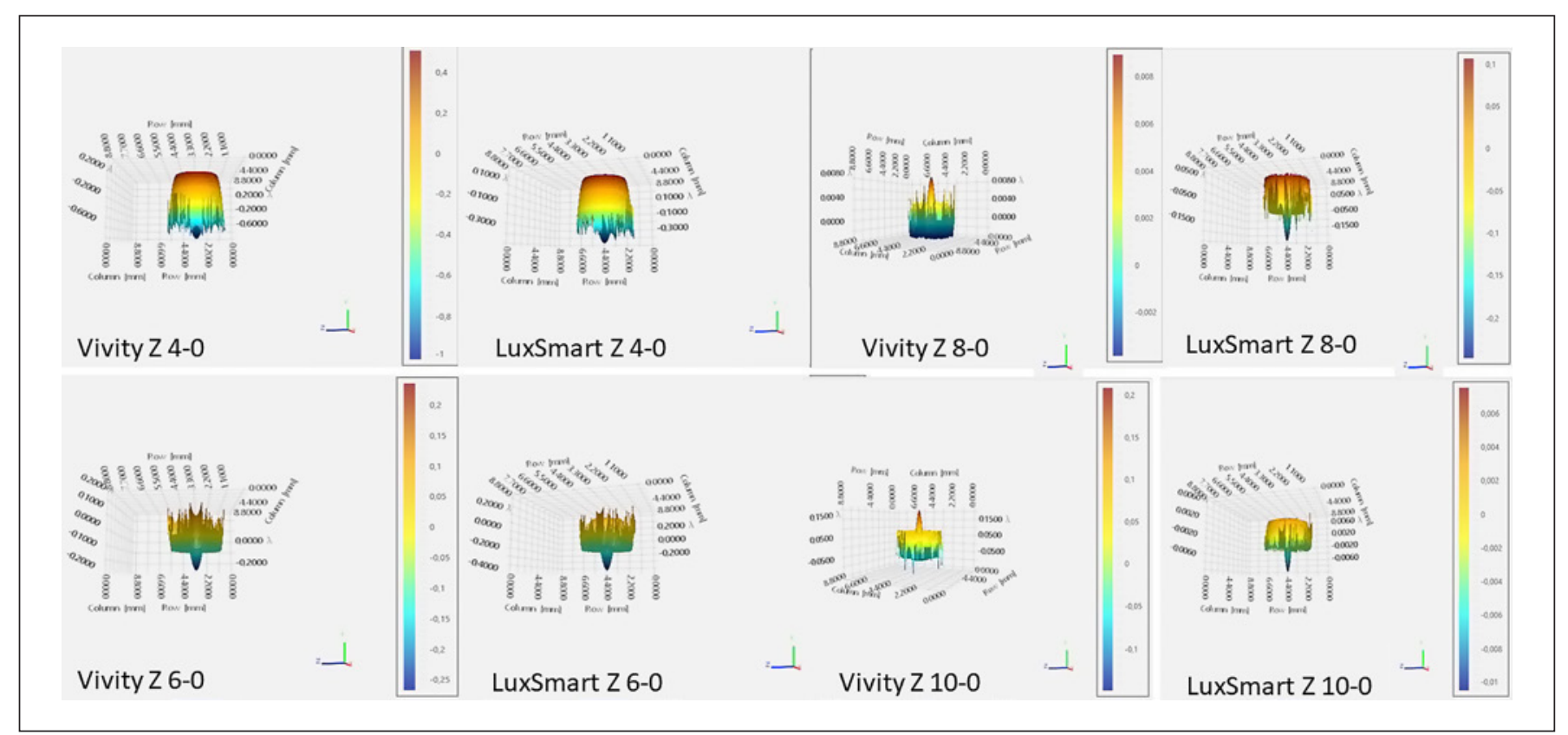

Fig. 6. Comparison of wavefront measurements with Z4-0, Z6-0, Z8-0, Z10-0.

more depth of field. Some additional lower order aberrations like astigmatism and HOAs like coma or trefoil may be most likely due to manufacturing errors of the lenses. Lenses showing an asymmetric wavefront mapping were therefore measured again up to 3 times. Wavefront errors other than SA could be reproduced in detail and thus may be caused by minor manufacturing errors. The central part of Vivity IOL showed a very pronounced negative SA 4-0 of $-1.01 \lambda$ combined with an SA 6-0 of $0.27 \lambda$ and an SA $10-0$ of $-0.21 \lambda$ in an apparently sinusoidal central profile pattern. Peripheral to this more extended central part of the IOL of about $2 \mathrm{~mm}$, after a very small transitional zone, the aberration values were measured positive, corresponding to the aberration correcting design of this lens platform. Vivity IOL's amplitude of aberrations was larger than that of LuxSmart. The LuxSmart IOL showed a slightly different and even more complex wavefront mapping than the Vivity, and the HOA modulation appeared to be more restricted to the center of the optic. The very central zone was $<1.5 \mathrm{~mm}$, and the optics showed an increase in negative SA 4-0 of $-0.49 \lambda$, an increased positive SA 6-0 of $0.46 \lambda$, and additionally an increased negative SA $8-0$ of $-0.25 \lambda$. Whereas the inner mid-periphery of the optics had a pronounced ring of positive aberration values, the outer periphery of the lens was aberration neutral. The optic of
LuxSmart behaves as aberration neutral already from about $1.5 \mathrm{~mm}$ of eccentricity. Overall HOAs (peak-tovalley; PV) were largest in Vivity (1.73) and moderate in LuxSmart (1.47).

\section{Discussion}

IOLs have evolved tremendously in recent years. There are a variety of different lens designs and IOL models available from various manufacturers. Monofocal IOLs can provide excellent distance vision but require glasses to focus on the missing areas at near and intermediate distances. Multifocal IOLs, mostly diffractive optics, should provide the desired spectacle independence at far to near distance in order to further improve quality of life. Unfortunately, this advantage increased glare and halos and leads to loss of contrast. These disadvantages can have a negative impact on overall satisfaction, primarily when driving by night or in rainy weather, for example. In the past, numerous studies have been performed to analyze and compare the optical quality of different monofocal, multifocal, and EDoF IOLs. Unfortunately, in vitro measurement setups as well as clinical evaluations with reported results are often not directly comparable. Different manufacturers use different optical measuring 
principles. Therefore, 1 IOL may have better performance and results depending on the setting. A direct comparison between different lenses is often not given. It is obvious that due to different designs and materials, IOLs show very different optical behavior. For example, patients with a larger pupil may experience more optical phenomena such as halo, glare, or starburst.

In this in vitro study, 2 new EDoF IOLs with different designs were compared. According to the manufacturers, this new generation of IOLs will expand the lens portfolio for various fields of application. In the recent past, numerous clinical studies have been performed comparing light interference of trifocal IOLS and diffractive EDoF IOLs with extended depth of focus (EDOF). These studies were able to confirm that the trifocal IOLs were superior at near distance, while the EDOF IOLs performed better at mid-distance/intermediate distance. However, dysphotopsia occurred with all lens systems evaluated. EDOF IOLs are, according to current knowledge, preferable for patients who primarily work in the middle range (e.g., computer) and accept glasses for reading in close distance.

This analysis of new, innovative IOL optics based on increased negative SA should help the ophthalmic surgeon to select the IOL which, on the one hand, meets the individual requirements of the patient for functional vision without glasses and, on the other hand, best suits the individual eye. The measurements in this study comply with international standards and were carried out independently without financial support in order to provide an objective comparison between lenses from 2 different manufacturers. Understanding the principles of optical designs of new IOLs is imperative to understand the potential image quality and contrast transfer function. Our in vitro analysis on the optical bench must of course be confirmed and complemented by further clinical investigations with a larger number of patients.

\section{Conclusion}

The desire for postoperative spectacle independence with minimal risks and side effects, such as the development of dysphotopsia, is the main reason for the development of EDOF IOLs. In the last years, new lens models with different optical concepts were introduced into the competitive market. In order to keep an objective overview as a cataract or refractive surgeon and to meet the high demands of patients, a good basic knowledge of the different IOLs and their optical designs is necessary. It seems that there is no perfect IOL that is equally suitable for all patients, but the right choice is an individual, customized approach dealing with patients' expectations. The diversity of EDOF IOLs, their optics, and their respective impact on the vision quality must be understood in order to select the appropriate IOL in each individual case.

\section{Acknowledgments}

We would like to thank the company Trioptics which carried out all measurements according to highest scientific standards and ISO guidelines and provided us with official certificates for the measurements.

\section{Statement of Ethics}

The article is exempt from Ethical Committee approval as it is an optical bench analysis (in vitro study) without humans involved.

\section{Conflict of Interest Statement}

All authors have no financial or proprietary interest in any material or method mentioned.

\section{Funding Sources}

In this study, no sponsors were involved. There was no funding.

\section{Author Contributions}

A.F.B. and R.S. carried out the study design and wrote the manuscript. All authors discussed the results and contributed to the final manuscript.

\section{Data Availability Statement}

All data generated or analyzed during this study are included in this article. Further inquiries can be directed to the corresponding author. 


\section{References}

1 De Silva SR, Evans JR, Kirthi V, Ziaei M, Leyland M. Multifocal versus monofocal intraocular lenses after cataract extraction. Cochrane Database Syst Rev. 2016;12(12): CD003169.

2 Goemaere J, Trigaux C, Denissen L, Dragnea D, Hua MT, Tassignon MJ, et al. Fifteen years of IOL exchange: indications, outcomes, and complications. J Cataract Refract Surg. 2020 Dec;46(12):1596-603. PMID: 33259388.

3 Shajari M, Priglinger S, Kreutzer T. (Current state of knowledge on special lenses). Ophthalmologe. 2020 Mar;117(3):283-92. PMID: 32112219.

4 Böhm M, Petermann K, Hemkeppler E, Kohnen T. Defocus curves of 4 presbyopiacorrecting IOL designs: diffractive panfocal, diffractive trifocal, segmental refractive, and extended-depth-of-focus. J Cataract Refract Surg. 2019 Nov; 45(11):1625-36. PMID: 31706517.

5 Tandogan T, Son HS, Choi CY, Knorz MC, Auffarth GU, Khoramnia R. Laboratory evaluation of the influence of decentration and pupil size on the optical performance of a monofocal, bifocal, and trifocal intraocular lens. J Refract Surg. 2017 Dec 1;33(12):80812. PMID: 29227508

6 Ortiz C, Esteve-Taboada JJ, Belda-Salmerón L, Monsálvez-Romín D, Domínguez-Vicent A. Effect of decentration on the optical quality of two intraocular lenses. Optom Vis Sci. 2016 Dec;93(12):1552-9. PMID: 27776082.

7 Oltrup T, Bende T, Al-Mohamedi H, Cayless A, Bende M, Leitritz MA, et al. Comparison of spherical and aspherical intraocular lenses with decentration and tilt error using a physical model of human contrast vision and an image quality metric. Z Med Phys. 2021 Aug; 31(3):316-26. Epub ahead of print. PMID: 33612389 .

8 Escandón-García S, Ribeiro FJ, McAlinden C, Queirós A, González-Méijome JM. Throughfocus vision performance and light disturbances of 3 new intraocular lenses for presbyopia correction. J Ophthalmol. 2018 Jan 31; 2018:6165493. PMID: 29651343; PMCID: PMC5831879.

9 Breyer DRH, Beckers L, Ax T, Kaymak H, Klabe K, Kretz FTA. Aktuelle Übersicht: multifokale linsen und extended-depth-of-focusintraokularlinsen (current review: multifocal intraocular lenses and extended depth of focus intraocular lenses). Klin Monbl Augenheilkd. 2020 Aug;237(8):943-57. Epub 2020 Aug 14. PMID: 32797470.

10 Tran DB, Owyang A, Hwang J, Potvin R. Visual acuity, quality of vision, and patient-reported outcomes after bilateral implantation with a trifocal or extended depth of focus intraocular lens. Clin Ophthalmol. 2021 Feb 3; 15:403-12. PMID: 33568894; PMCID: PMC7868299.

11 Sudhir RR, Dey A, Bhattacharrya S, Bahulayan A. AcrySof IQ panoptix intraocular lens versus extended depth of focus intraocular lens and trifocal intraocular lens: a clinical overview. Asia Pac J Ophthalmol. 2019 JulAug;8(4):335-49. PMID: 31403494; PMCID: PMC6727923.

12 Auffarth GU, Gerl M, Tsai L, Janakiraman DP, Jackson B, Alarcon A, et al. Clinical eval- uation of a new monofocal intraocular lens with enhanced intermediate function in cataract patients. J Cataract Refract Surg. $2021 \mathrm{Feb}$ 1;47(2):184-91. PMID: 32932369.

13 Rocha KM, Vabre L, Chateau N, Krueger RR. Expanding depth of focus by modifying higher-order aberrations induced by an adaptive optics visual simulator. J Cataract Refract Surg. 2009;35:1885-92.

14 Cochener B, Boutillier G, Lamard M, Auberger-Zagnoli C. A comparative evaluation of a new generation of diffractive trifocal and extended depth of focus intraocular lenses. J Refract Surg. 2018 Aug 1;34(8):507-14. PMID: 30089179.

15 Schallhorn JM. Multifocal and extended depth of focus intraocular lenses: a comparison of data from the United States food and drug administration premarket approval trials. J Refract Surg. 2021 Feb 1;37(2):98-104. PMID: 33577695.

16 MacRae S, Holladay JT, Glasser A, Calogero D, Hilmantel G, Masket S, et al. Special report: American academy of ophthalmology task force consensus statement for extended depth of focus intraocular lenses. Ophthalmology. 2017;124:139-41.

17 Food and Drug Administration. Summary of Safety and Effectiveness Data. AcrySofTM IQ VivityTM Extended Vision Intraocular Lens. 2020;PMA P930014/S126.

18 Norrby NE, Grossman LW, Geraghty EP, Kreiner CF, Mihori M, Patel AS, et al. Determining the imaging quality of intraocular lenses. J Cataract Refract Surg. 1998;24(5): 703-14. 